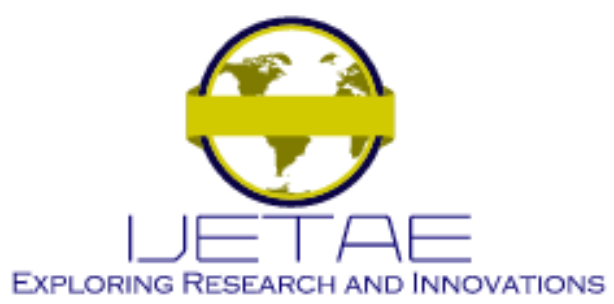

International Journal of Emerging Technology and Advanced Engineering

Website: www.ijetae.com (E-ISSN 2250-2459, Scopus Indexed, ISO 9001:2008 Certified Journal, Volume 12, Issue 02, February 2022)

\title{
Comparative Performance Analysis of Real-Time Methods for Cassava Phytoplasma Disease (CPD) Detection based on Deep Learning Neural Networks
}

\author{
Irma T. Plata ${ }^{1}$, Edward B. Panganiban ${ }^{2}$, Darios B. Alado ${ }^{3}$, Allan C. Taracatac ${ }^{4}$, Bryan B. Bartolome ${ }^{5}$, \\ Freddie Rick E. Labuanan ${ }^{6}$ \\ ${ }_{1,2,3,4,5,6}$ CCSICT, Isabela State University, San Fabian, Echague, Isabela, Philippines
}

\begin{abstract}
Cassava Phytoplasma Disease (CPD) is a crop disease that reduces cassava output and quality. As a result, detection is essential in precision agriculture. On the greater area of the cassava field, manual identification of CPD illnesses takes more time and effort. Convolutional Neural Networks (CNN), a deep learning method, may be used to detect illnesses on leaves and other sections of cassava plants with greater accuracy. The approaches utilized in this study assisted in the identification of CPD by completing customized training/fine-tuning on three CNN models for object recognition: Faster R-CNN, SSD Mobilenet v2, and YOLO v4. The Faster R-CNN inception v2 has a 95 percent training accuracy, SSD Mobilenet $v 2$ has a 73 percent training accuracy, and YOLOv4 has an 85 percent training accuracy, according to the data. Finally, the study found that the YOLOv4 outperforms the Faster R-CNN inception v2 and SSD MobileNet $v 2$ in terms of image computing capacity. However, Faster R-CNN inception v2 performs the best compared to the two other models in terms of accuracy. Hence, these two models can be used depending on the purpose of CPD detection. However, since CPD detection is the main purpose of this study, the Faster R-CNN model is recommended for adoption to detect $\mathrm{CPD}$ in a real-time environment.
\end{abstract}

Keywords - cassava phytoplasma disease, convolutional neural networks, faster $\mathrm{R}-\mathrm{CNN}$, image processing, precision agriculture.

\section{INTRODUCTION}

In the field of computer vision, object detection has been facing a fast revolution. Its involvement in both object categorization and object location makes it one of the most difficult applications in the computer vision area. To put it another way, this detection approach seeks to figure out where items are in a given image and which category they belong to.
In this study, the object classification was involved in detecting the presence of phytoplasma diseases in cassava farms located in San Guillermo, Isabela, which was chosen as the testing site. However, it is necessary to research which real-time object detection technology will be employed and which new and inventive strategies will be used in the process. As a result, some strategies for improving object detection performance have been presented.

For context, the convolutional neural network $(\mathrm{CNN})$ is an effective deep learning model that incorporates hierarchical learning features. According to research, CNNextracted features have a higher discriminating and generalization capacity than hand-crafted features [1]. CNN has had a lot of success in the field of computer vision. Furthermore, deep learning may produce greater precision and reduce test time [2].

Crop diseases like the cassava Phytoplasma diseases (CPD) play a key role in reducing cassava production and quality. Therefore, detection is fundamental in precision agriculture tasks. Manual detection of phytoplasma diseases takes additional time and effort on the larger area of the cassava farm. A deep learning approach like the CNN can be used to detect diseases more accurately on leaves and other parts of the cassava plants.

The study's general objective is to analyze real-time methods for CPD detection through comparative performance analysis. The involved methods in this study helped detect CPD diseases. In the process, it performs three CNN models for object detection comprising Faster R-CNN, SSD Mobilenet v2, and the YOLO v4. 


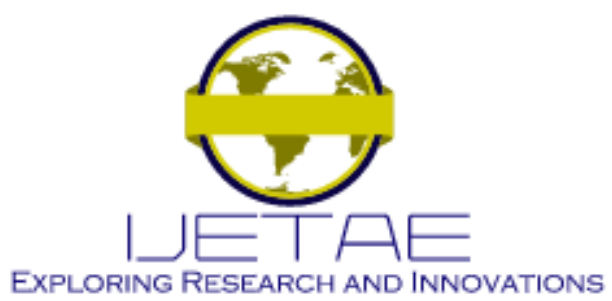

International Journal of Emerging Technology and Advanced Engineering Website: www.ijetae.com (E-ISSN 2250-2459, Scopus Indexed, ISO 9001:2008 Certified Journal, Volume 12, Issue 02, February 2022)

\section{THEORETICAL Framework Of THE StUdy}

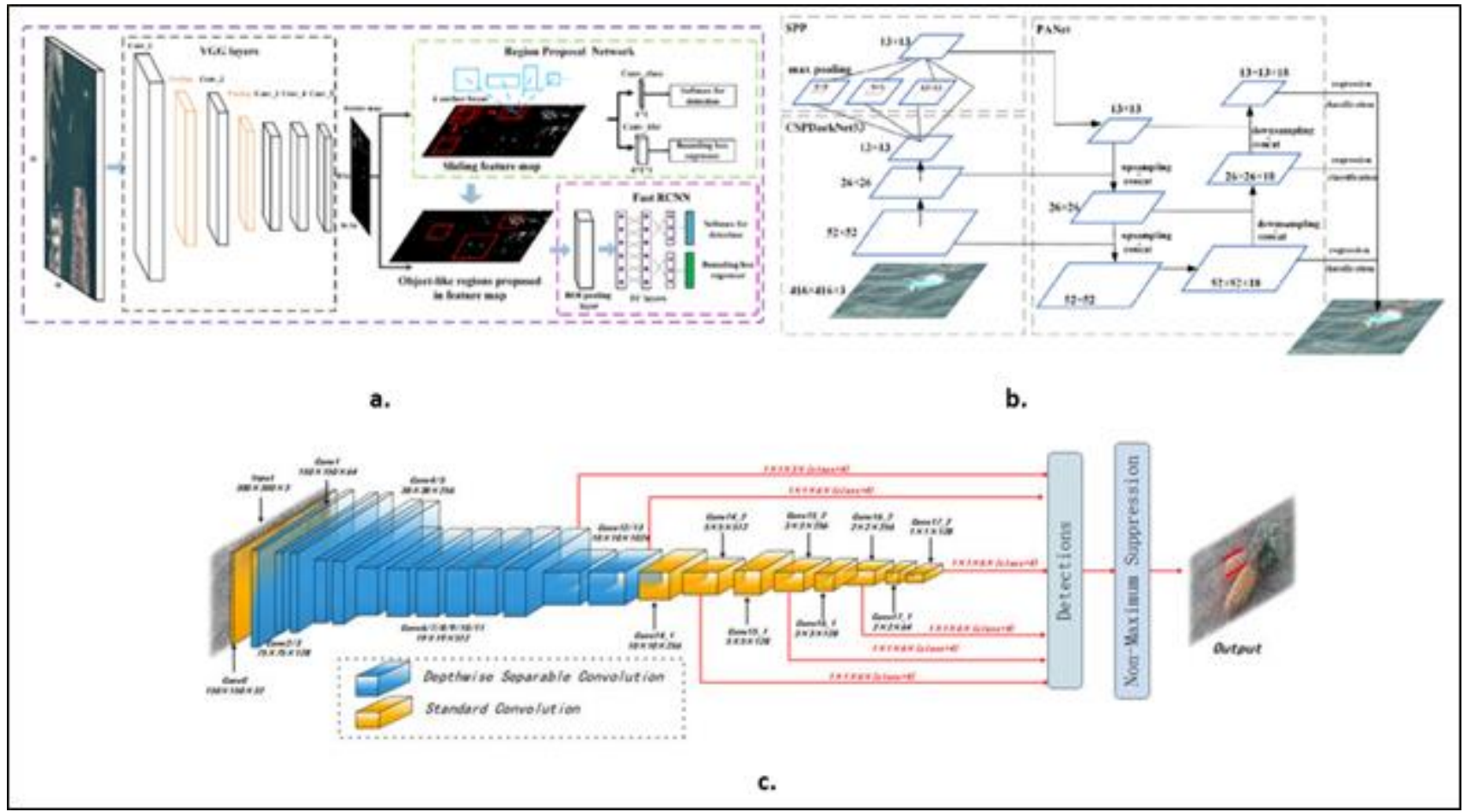

Figure 1. Real-time CPD detection and quantification Framework Model

The study was designed based on the theories of deep learning frameworks. At the writing of this study, the Faster R-CNN, SSD Mobilenet v2, and YOLO v4 were among the best performing and widely used deep learning frameworks for real-time object detection applications for their speed and accuracy presented in Figure 1. Detailed methods and procedures behind each framework entail the following:

\section{Faster R-CNN Inception $v 2$}

The R-CNN [6], which utilizes search selectivity [1] to locate areas of interest and feeds them to a ConvNet, is the initial stage in Faster R-CNN. By grouping comparable pixels and textures into numerous rectangular boxes, it tries to discover areas that could constitute an item. The R-CNN article uses two thousand recommended regions (rectangular boxes) from search selection. The 2,000 locations are then fed into a CNN model that has already been trained.
Finally, the outputs (feature maps) are fed into an SVM, classifying them. The regression between predicted and ground-truth bounding boxes (bboxes) is calculated.

The Fast R-CNN [7] advances one step. Instead of applying $\mathrm{CNN}$ to recommended areas 2,000 times, the original image is simply sent to a pre-trained CNN model once. The output feature map of the previous stage is used to calculate the search selection algorithm.

The ROI pooling layer is then utilized to verify that the output size is standard and pre-defined. As inputs, these acceptable outputs are passed to a fully linked layer. Finally, a softmax classifier is utilized to predict the observed item, and a linear regressor is used to modify bounding box localizations.

Figure 2 shows that the Faster R-CNN [8] (frcnn for short) makes more progress than the Fast R-CNN. The Region Proposal Network (RPN) replaces the search selection process. RPN is a network that proposes regions, as the name implies. 


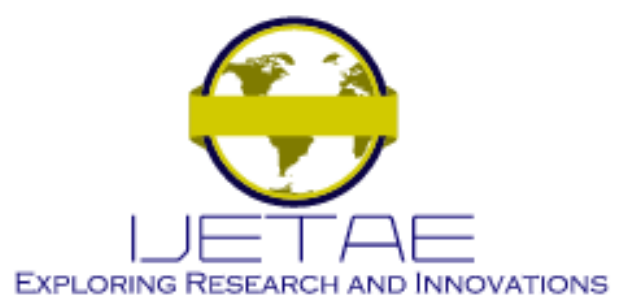

International Journal of Emerging Technology and Advanced Engineering

Website: www.ijetae.com (E-ISSN 2250-2459, Scopus Indexed, ISO 9001:2008 Certified Journal, Volume 12, Issue 02, February 2022)

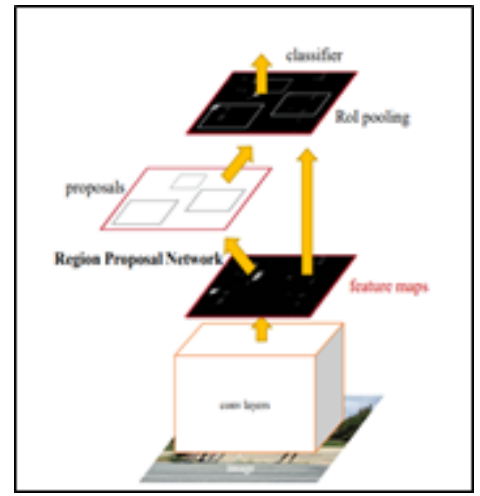

Figure 2. Faster R-CNN is a single, unified network for object detection [8]

Figure 3 shows the Region Proposal Network (RPN), a simple neural network that slides on the last feature map of the convolution layers and predicts whether or not there is an item and the bounding box of such things.

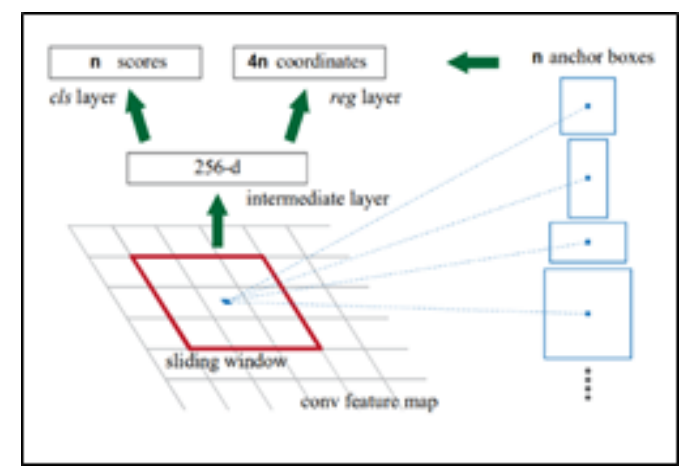

Figure 3. Faster R-CNN: Region Proposal Network

\section{SSD Mobilenet v2 MSCOCO}

Figure 4 shows the single-shot detection (SSD) architecture, which is a single convolutional network that learns to anticipate bounding box positions and categorize them in one run. As a result, SSD may be trained from beginning to end. The SSD network is made up of a basic architecture (in this case, MobileNet) and numerous convolution layers:

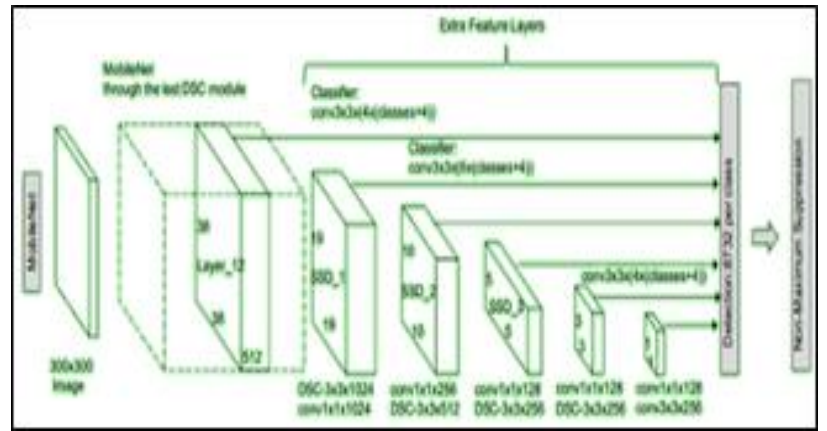

Figure 4. SSD Mobilenet Layered Architecture [9]

Using SSD only needs to take one single shot to detect multiple objects within the image. At the same time, regional proposal network (RPN) based approaches such as the R-CNN series need two shots, one for generating region proposals one for detecting the object of each proposal. Thus, SSD is faster compared with two-shot RPN-based approaches.

\section{YOLOv4}

Figure 5 shows YOLOv4 [2], which was touted to be one of the most advanced real-time object detectors at the time. In comparison to YOLOv3, YOLOv4 is 12 percent speedier and $10 \%$ more accurate, according to the report.

The new YOLOV4 design uses CSPDarknet54 as a backbone, enhancing CNN's learning capabilities. Furthermore, general features such as Weighted-ResidualConnections (WRC), Cross-Stage-Partial-Connections (CSP), Cross mini-Batch Normalization (CmBN), Selfadversarial-training (SAT), and Mish-activation aid YOLOv4 in achieving outstanding outcomes.

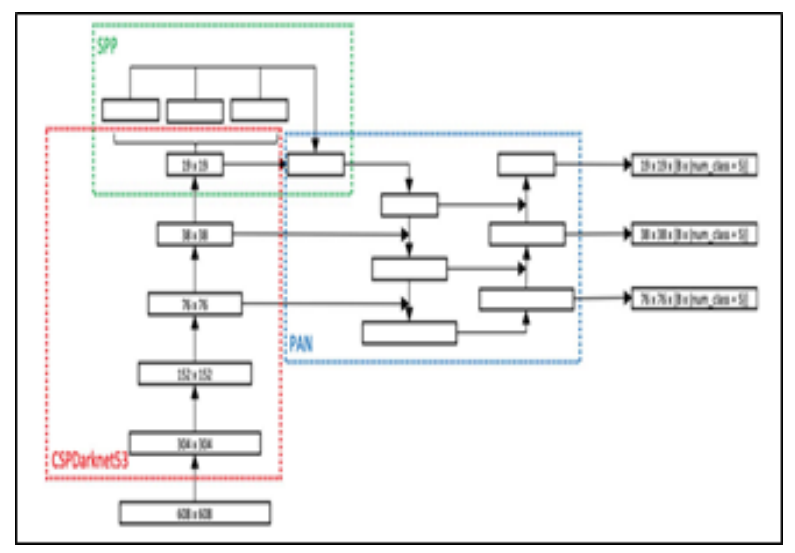

Figure 5. YOLOv4 model architecture [10] 


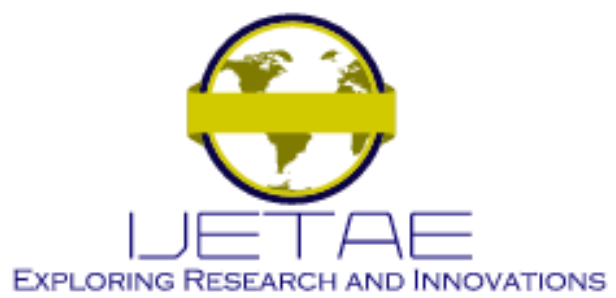

International Journal of Emerging Technology and Advanced Engineering

Website: www.ijetae.com (E-ISSN 2250-2459, Scopus Indexed, ISO 9001:2008 Certified Journal, Volume 12, Issue 02, February 2022)

\section{Methodology}

To adopt each CNN model for custom CPD real-time detection, we have performed the following procedures:

1. Datasets preparation and collection of datasets for training process

2. Perform the pre-processing procedures for collected datasets

3. Train the datasets and perform fine-tuning using the three neural models

4. Optimize the three neural network models

5. Compare the performance of the three neural network models in determining the best real-time detecting technique for CPD.

\section{Datasets preparation and collection for training process}

Images of cassava showing physical CPD signs, ranging in age from three to six months, were used to create the image datasets, which a plant pathologist then validated. The datasets were collected in multiple site locations with an identified incidence of cassava phytoplasma disease (CPD) with the assistance of a plant pathologist and the LGU-MOA of San Guillermo. Severe CPD infections were spotted at Villa Teresita, San Guillermo, Isabela, and at Banwar, Cabarroguis, Quirino. Cassava severely affected varieties are Rayong 5, Lakan, and KU 50.

The images of CPD infected cassavas were taken using the improvised overhead camera mount, between 10:00 AM to 3:00 PM, across the different cassava varieties and ages at different heights and angles with minimal leaves overlapping, further diversifying the datasets. A total of 40,360 images were extracted and used. $80 \%$ of all images were the train datasets, while the other $20 \%$ were the test datasets.

In the absence of the actual drone to collect datasets, a three-meter long PVC pipe and a 16 mega-pixels NDVI camera facing down with an infra-red (IR) sensitivity rate of 1200 nanometers and a built-in red filter installed at the other end of the PVC pipe was used as presented in Figure 6.

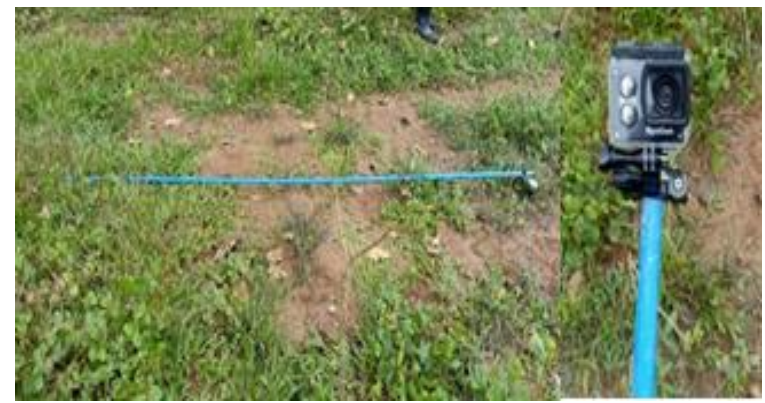

Figure 6. Improvised overhead camera mount
This procedure mimics the drone taking overhead images at different viewing angles as it passes through. The camera installed was also set to record continuous video footage of the scene considering all the external factors such as camera movements, vibrations, sudden altitude change, wind velocity, light condition (sunny/cloudy) that may significantly affect the video quality. These factors commonly occur in open-field settings and work naturally as white-noises to our datasets to further augment its ability to recognize CPD infected cassava at different environmental conditions, as illustrated in Figures 7,8, and 9.

\section{Video and Image processing tools and technologies}

Computer Vision Annotation Tool (CVAT) is an opensource tool for annotating digital images and videos. The application's primary function is to provide users with convenient annotation instruments.

CVAT is a browser-based application for individuals and teams that supports different work scenarios. The main tasks of supervised machine learning can be divided into three groups:

1. Object detection

2. Image classification

3. Image segmentation

CVAT allows you to annotate data for each of these cases.

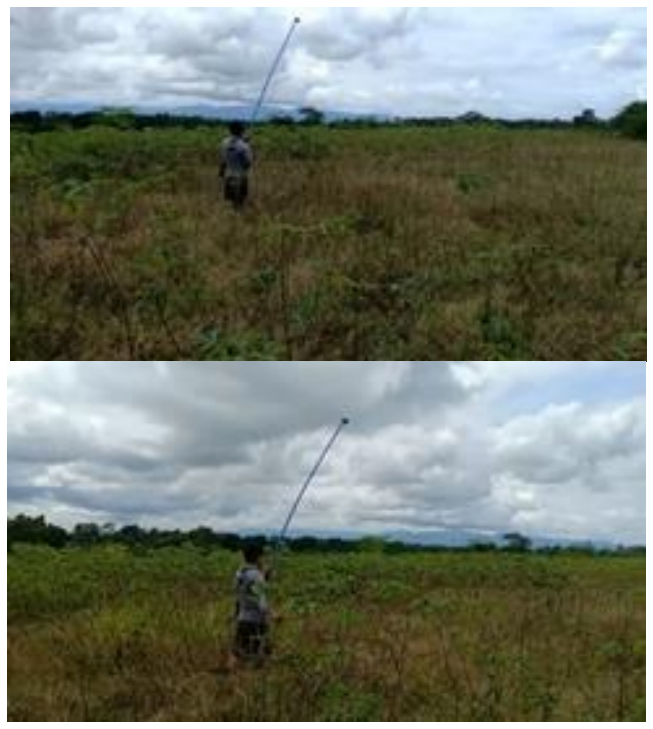

Figure 7. Actual activity during the data gathering for CPD image datasets 


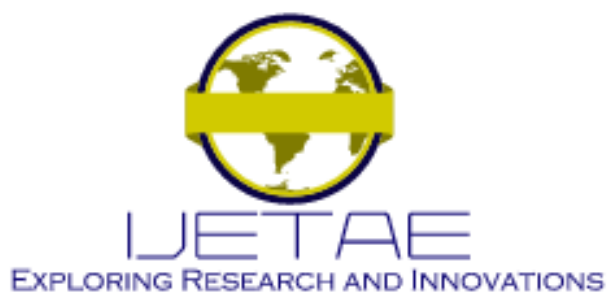

International Journal of Emerging Technology and Advanced Engineering

Website: www.ijetae.com (E-ISSN 2250-2459, Scopus Indexed, ISO 9001:2008 Certified Journal, Volume 12, Issue 02, February 2022)

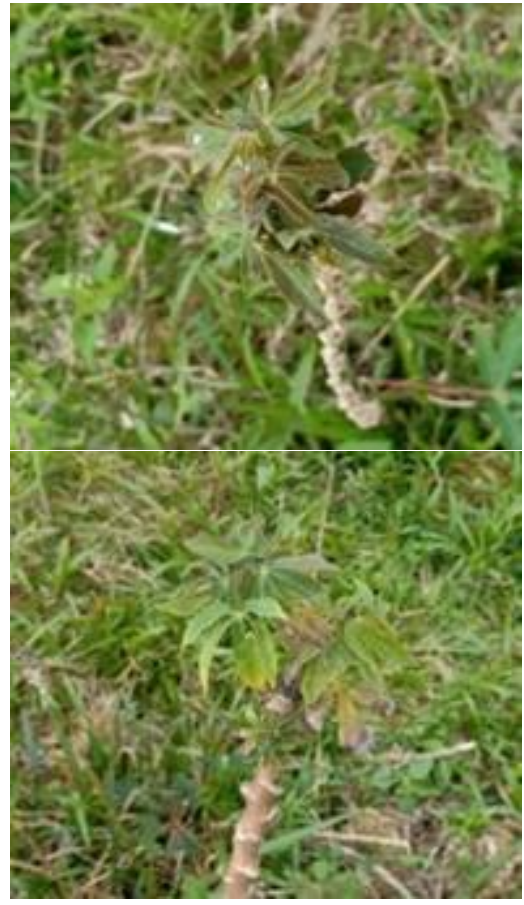

Figure 8. Sample RGB images of CPD infected cassava that manifests physical symptoms
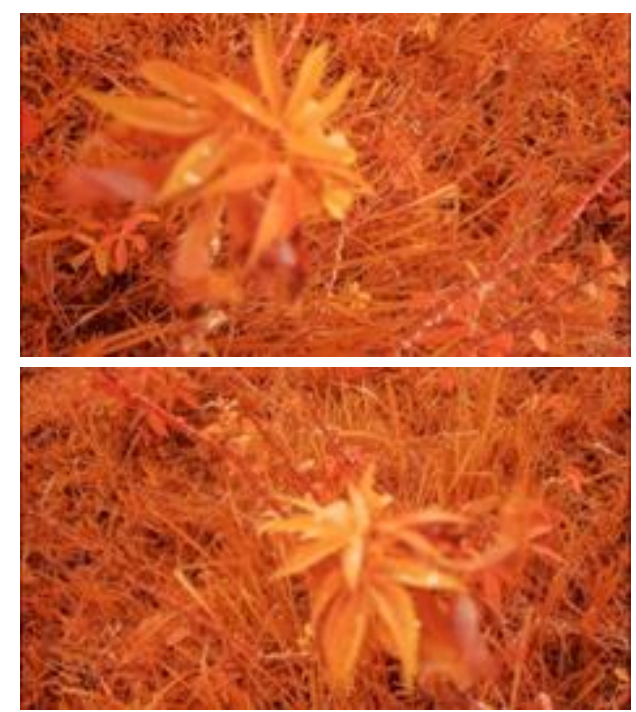

Figure 9. Sample raw NDVI images of CPD infected cassava that manifests physical symptoms

Performing the pre-processing procedures for collected datasets

The raw IR video footage is preprocessed using our developed application to take a raw IR video file as an input.
Automatically calculate its NDVI value per pixel based on the equation presented in Figure 10 and render it on a new video file [11] as visualized in Figure 11.).

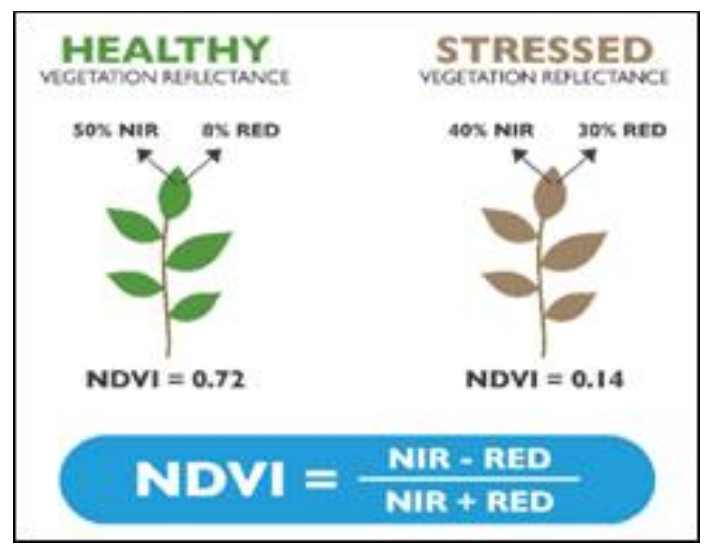

Figure 10. Sample computed NDVI value

(https://www.agricolus.com/en/indici-vegetazione-ndvi-ndmi istruzioni-luso/)

These rendered video files with the calculated NDVI values are then converted into images per frame. Thus, generating several thousands of high-quality CPD images.
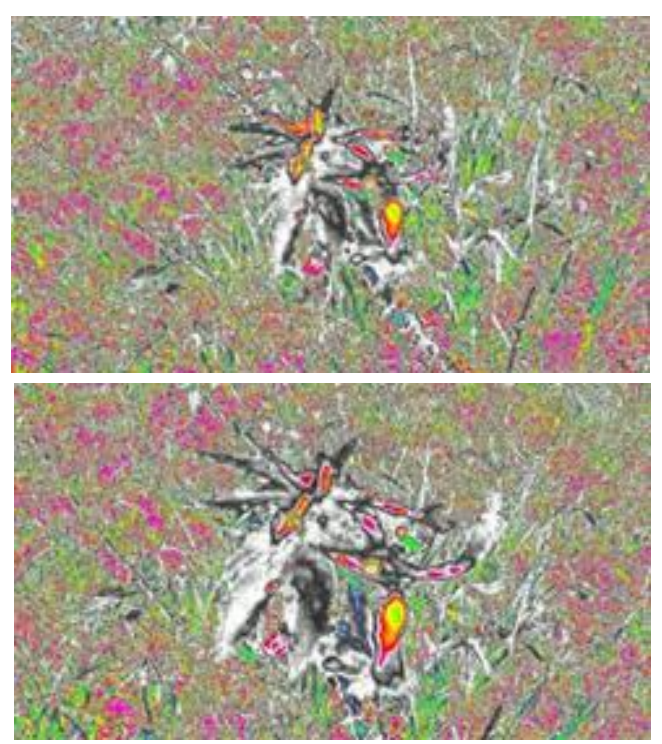

Figure 11. Visualization of calculated NDVI values from a CPD infected cassava that manifests physical symptoms

A total of not less than 75,000 frames when all the rendered video files of calculated NDVIs were combined, excluding the removed scenes that do not include our subject. 


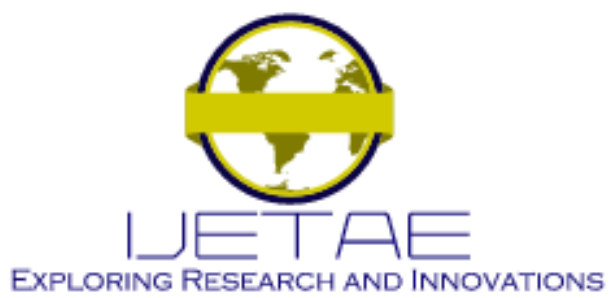

International Journal of Emerging Technology and Advanced Engineering Website: www.ijetae.com (E-ISSN 2250-2459, Scopus Indexed, ISO 9001:2008 Certified Journal, Volume 12, Issue 02, February 2022)

\section{On Image annotation}

The rendered NDVI video files are uploaded into an open-source annotating tool. This tool automatically extracts images per frame and enables us to locate and specify using bounding boxes with our subject from the cluttered image. The bounding boxes determine each of our subjects' $\mathrm{x}$ and $\mathrm{y}$ coordinates on the image, as shown in Figure 12.

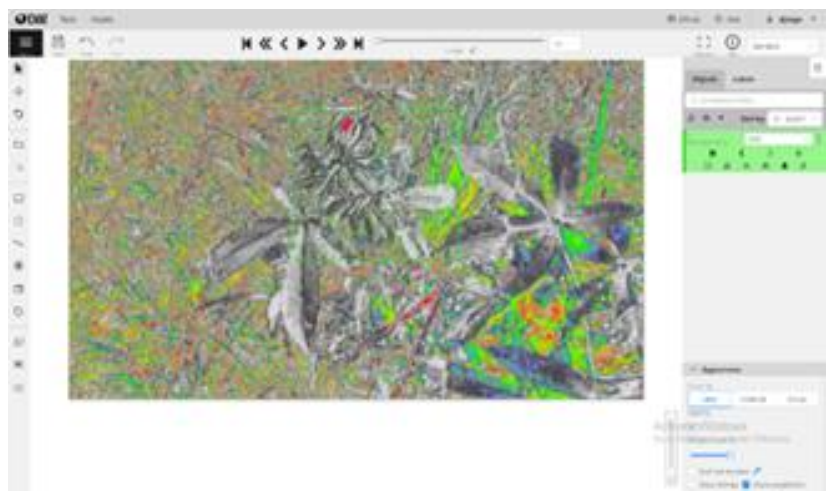

Figure 12. Open-source video/image annotating tool; Open VINOTM

After a thorough process of annotating each video frame containing our subject, roughly 50,000 frames/images were identified. After this process, the annotation is exported into TensorFlow record and YOLOv4 format. These now serve as our CPD datasets covering CPD infected cassava with visual symptoms ages from three to six months.

Training the datasets and performing fine-tuning using the three neural models

\section{Training/Fine-tuning model}

To fine-tune our deep learning models, we implemented a transfer learning approach by adapting an existing classifier (Faster R-CNN Inception v2 and SSD Mobilenet v2) to a custom task: detecting CPD infected cassava in real-time. The basic principle of transfer is that knowledge obtained while learning to do one activity may be used to aid increase learning performance in a related but unrelated one [12]. If done successfully, it would greatly improve the performance of learning [13].
With the transfer learning approach, we have developed a software application for semi-unsupervised deep learning with the integration of a rolling learning rate (LR) search cross-validation algorithm for custom object detection, as shown in Figure 13.

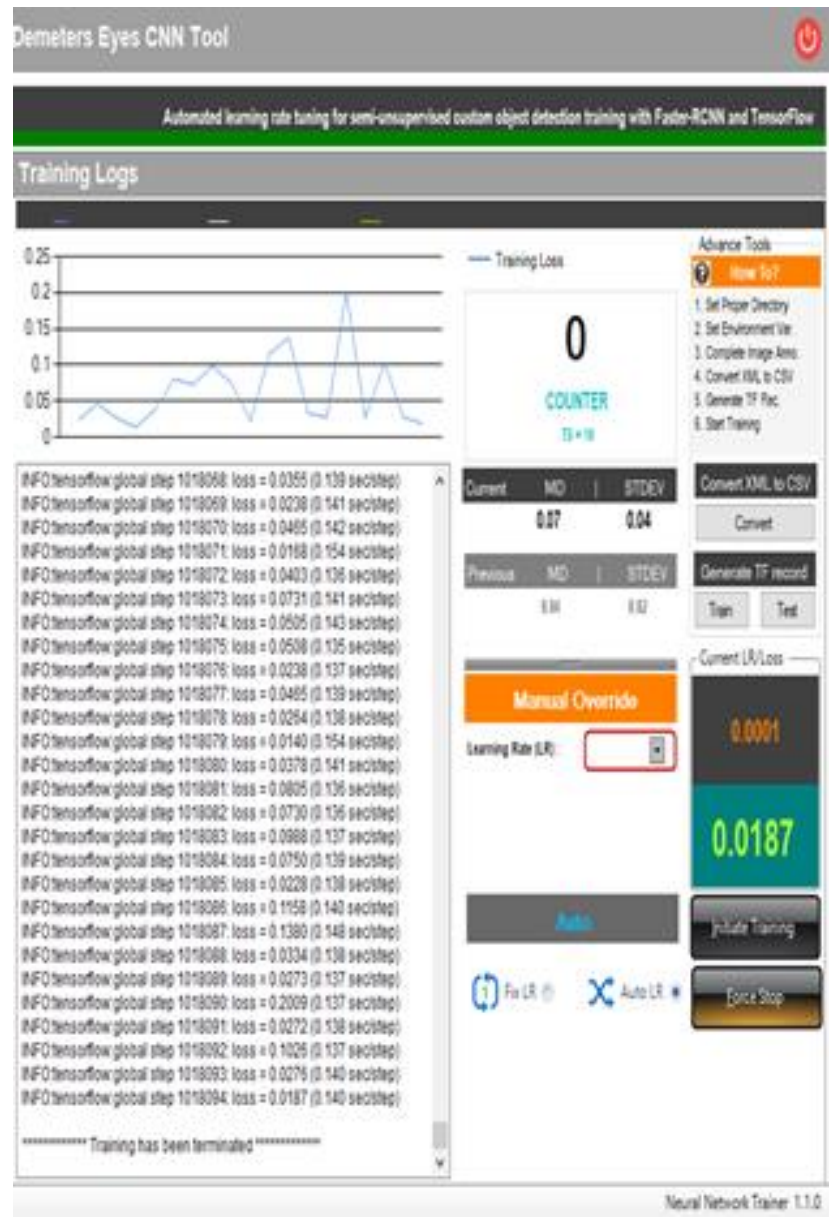

Figure 13. UI of the developed semi-unsupervised deep learning utility application 


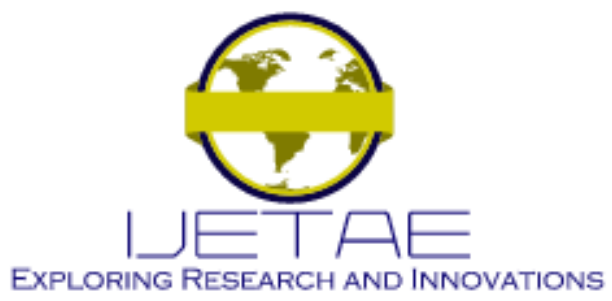

International Journal of Emerging Technology and Advanced Engineering

Website: www.ijetae.com (E-ISSN 2250-2459, Scopus Indexed, ISO 9001:2008 Certified Journal, Volume 12, Issue 02, February 2022)

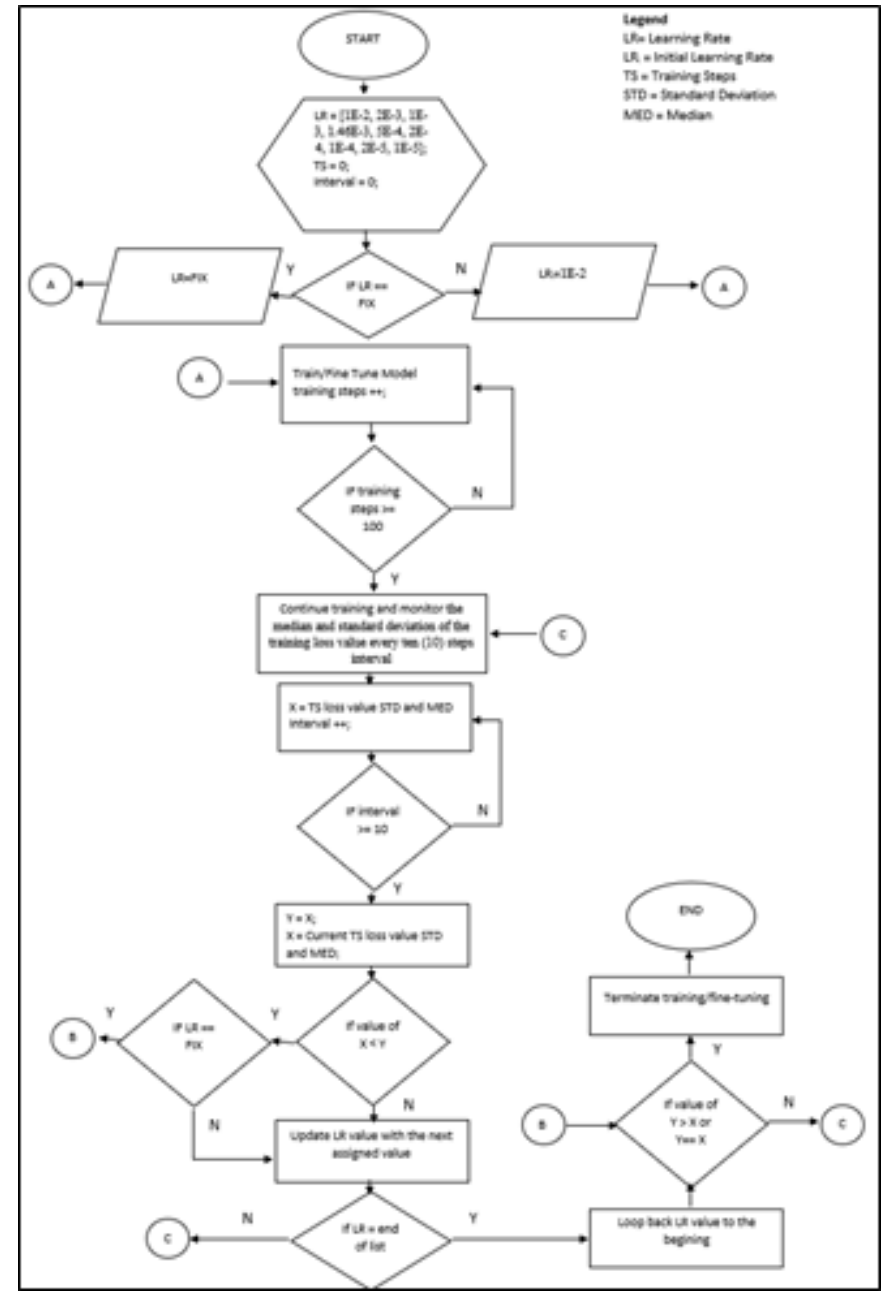

Figure 14. Semi-unsupervised deep learning utility application flowchart

The developed semi-unsupervised deep learning utility application process flow starts by either setting up a typical fixed learning rate or via a rolling learning rate search cross-validation (RLRSC-V) algorithm, illustrated in Figure 14. Using the RLRSC-V; to get the optimal LR value, the algorithm picks an initial LR $\{0.01\}$ from a list of pre-defined LR's (based on known ideal LR values used from different deep learning models), which are arranged in descending or in an array $\{1 \mathrm{E}-2,2 \mathrm{E}-3,1 \mathrm{E}-3,1.46 \mathrm{E}-3,5 \mathrm{E}-$ 4, 2E-4, 1E-4, 2E-5, 1E-5 .

Using the initial LR, the training will proceed until it reaches the 100th step. After reaching the 100th step, the algorithm will start monitoring the median and standard deviation of the training loss value for every ten (10) steps.
Furthermore, this process takes the value of the median. Standard deviation from the first ten (10) training steps after reaching the 100th step and stores its values in a public variable "X." After passing the succeeding ten (10) steps after the previous recorded median and standard deviation, the values from the variable " $X$ " will be moved to a variable " $Y$ " and the new values from the running median and standard deviation stores in the variable "X."

The algorithm will compare its values after completing the first cycle of passing values for the running median and standard deviation. If the median from the " $\mathrm{X}$ " variable is lower than the median value from the "Y" variable and the standard deviation from the " $\mathrm{X}$ " variable is less than one (1). The training will continue using the current LR value, else the training is paused, and the LR value is updated with the next value from the array list before resuming.

The algorithm is also designed to loop back into the first LR value from the array list if it reaches the end. This method is applied throughout the training process until it finds the most optimal LR that maintains the pattern of descending loss value. The program was set to automatically terminate the training/fine-tuning if it detects no significant improvements or if the trend of loss value is continuously ascending.

To assess the performance of the developed application, we have simulated a transfer learning session using Faster R-CNN Inception v2 as our base model. We used a total of 4,088 image datasets with one (1) class. Eighty percent of the images were used for training and 20 percent for the test set. The initial LR value for both algorithms is set to 0.0001(default LR). The loss values of both algorithms are recorded and compared for the first 500 training steps.

On the other hand, adapting the YOLO v4 architecture, we cloned the Github repository of https:/github.com/AlexeyAB/darknet and used convolutional weights that are pre-trained on Imagenet from the YOLOv4 model zoo, COCO pre-trained files $(512 \times 512)$. On the configuration file of YOLOv4, we have set 70 percent of our image dataset for training, 20 percent for testing, and 10 percent for validation. We also set the training batch size to 64 and subdivisions of 8. Other settings are retained as it is.

The machine used for training/fine-tuning all the deep learning models is powered by an Intel Core i9 8th Generation, 32GB DDR4 3600Mhz RAM, 1TB NVMe PCIe SSD, and an RTX 2080 Super 8GB. Furthermore, we installed TensorFlow GPU 1.14 and rebuilt OpenCV GPU 4.4 from the source using CMAKE software. 


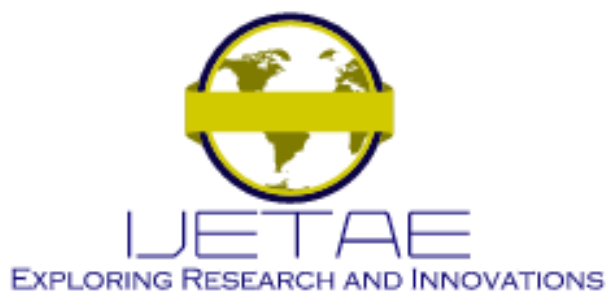

International Journal of Emerging Technology and Advanced Engineering

Website: www.ijetae.com (E-ISSN 2250-2459, Scopus Indexed, ISO 9001:2008 Certified Journal, Volume 12, Issue 02, February 2022)

Optimize the three neural network models

Model optimization

We set the score_threshold $=0.3 / 0.5$ (default is 0.0 ) before freezing/exporting the model to optimize the Faster $\mathrm{R}-\mathrm{CNN}$ model. We also removed parts of the frozen graph that are only needed for training, such as checkpoint saving; stripped out parts of the graph that are never reached; removed debug operations like CheckNumerics; folded batch normalization ops into the pre-calculated weights; and fused common operations into unified versions to help reduce the amount of computation needed when the network is only used for inference.

Compare the performance of the three neural network models in determining the best real-time detecting technique for CPD

\section{Model comparison}

To benchmark the performance of these three CNN models in terms of detection speed and accuracy, we tested each model on a simulated moving overhead camera shot. This allows us to observe and measure how each model performs.

\section{RESUlTS AND DisCUSSION}

Based on the tabulated results of the testing and evaluation conducted using the developed semi- unsupervised deep learning utility application as presented in Figures 15 and Figure 16, the typical fixed LR scored a loss value of 1.04 in the 500th training step while RLRSC$\mathrm{V}$ scored 0.88. This shows a significant margin of difference between the two algorithms. RLRSC-V scores the lowest loss value and displays a smooth descend trend compared with fixed LR. RLRSC-V was able to prevent and manage sudden spikes on loss value during the training session.

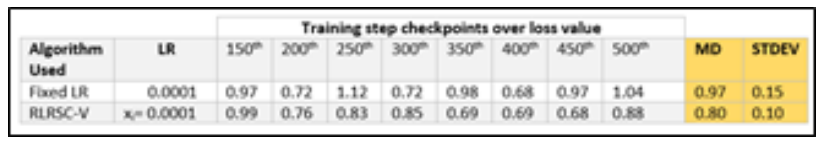

Figure 15. The tabulated result using fixed LR vs. RLRSC-V

\section{Loss Value Comparison}

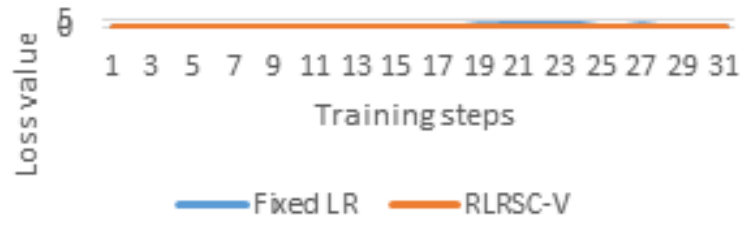

Figure 16. A snapshot from the loss value trend, fixed LR vs. RLRSC-V

Table 1.

Comparison of the three custom trained CNN models in terms of speed and accuracy

\begin{tabular}{|c|c|c|c|c|c|c|}
\hline & CNN Model & $\begin{array}{c}\text { Training } \\
\text { Datasets }\end{array}$ & $\begin{array}{c}\text { Test } \\
\text { Datasets }\end{array}$ & $\begin{array}{c}\text { Average Image } \\
\text { processing speed }\end{array}$ & $\begin{array}{c}\text { Training } \\
\text { Loss }\end{array}$ & $\begin{array}{c}\text { Training } \\
\text { Accuracy }\end{array}$ \\
\hline 1 & $\begin{array}{c}\text { Faster R-CNN } \\
\text { inception v2 }\end{array}$ & 40,000 & 10,000 & $11 \mathrm{fps}$ & $=0.05$ & $95 \%$ \\
\hline 2 & $\begin{array}{c}\text { SSD Mobilenet v2 } \\
\text { COCO }\end{array}$ & 40,000 & 10,000 & $20 \mathrm{fps}$ & $=0.27$ & $73 \%$ \\
\hline 3 & YOLOv4 & 40,000 & 10,000 & $25 \mathrm{fps}$ & $=0.15$ & $85 \%$ \\
\hline
\end{tabular}

With this encouraging result, we used the RLRSC-V program to fine-tune our proprietary CPD detection model based on Faster R-CNN Inception v2 and SSD Mobilenet $\mathrm{v} 2$ architecture. The remaining $20 \%$ of the picture dataset is used as the test set, with the remaining $80 \%$ used for training. With a minimum input picture dimension of 600 pixels and a maximum dimension of 1024 pixels, the training batch size was set to the default value of 1 .
After fine-tuning our custom CPD detection model, with the hardware specification that we have on our windows machine, the Faster R-CNN inception v2 deep learning model has an average image processing speed of 8 frames per second (FPS) by default using both CPU and GPU resources. Later on, we discovered that non-max suppression (NMS) computations are performed entirely on the CPU rather than on the GPU, which can be slower. The model we're using has a score threshold of zero, which means we never prune any predictions before performing non-max suppression. 


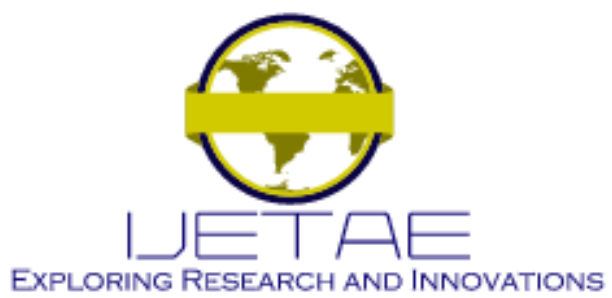

International Journal of Emerging Technology and Advanced Engineering

Website: www.ijetae.com (E-ISSN 2250-2459, Scopus Indexed, ISO 9001:2008 Certified Journal, Volume 12, Issue 02, February 2022)

Each model varies in terms of training duration, as illustrated in Figure 17. The Faster R-CNN inception v2 model took 53 hours to complete, the SSD Mobilenet v2 COCO model took 56 hours, and the YOLOv4 required just 17 hours to achieve its optimum detection accuracy.

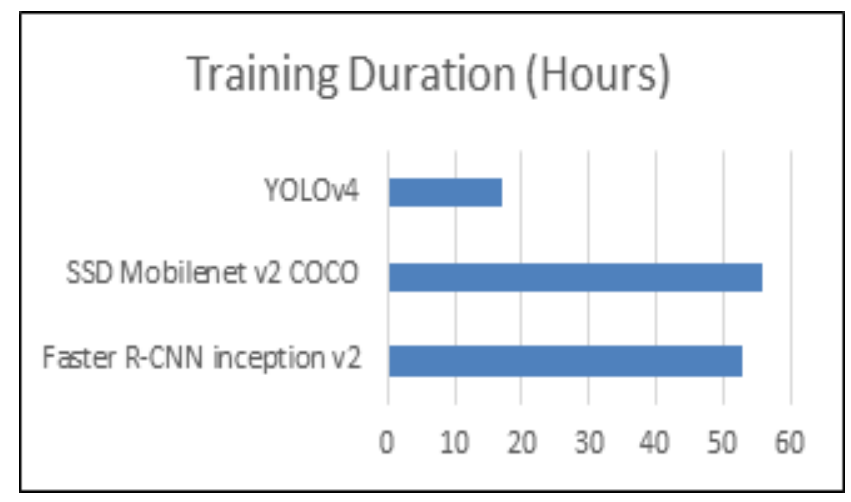

Figure 17. Comparison of the three (3) custom trained CNN models in terms of training time duration

All three custom-trained CNN models were optimized to minimize the workload on the computing machine and improve the overall image processing time. Based on Table 1 , faster R-CNN inception $\mathrm{v} 2$ has less than or equal to 0.05 training loss value with an average image processing speed of 11 FPS and training accuracy of 95\%. SSD Mobilenet v2 has greater than or equal to 0.27 training loss value with an average image processing speed of 20 FPS and training accuracy of $73 \%$. Finally, YOLOv4 has 85 percent training accuracy with an average image processing speed of 25 FPS and a training loss of 0.15 .

The image processing presented in the table involves onthe-fly calculation of NDVI values on each frame of the incoming live camera feed, followed by real-time CPD detection and quantification. From the table, if the consideration is the image processing speed, YOLOv4 is better than other models, but if the accuracy is the consideration, Faster R-CNN inception v2 performs better. Hence, these two models can be used depending on the purpose of the detection of the CPD. However, the most important factor to be considered must be its accuracy since CPD detection is the main objective of this study.

\section{Limitations}

The wireless digital video transmission only works effectively within a maximum radius of two (2) kilometers in an open field using the defined equipment.
In addition, radio signal obstacles such as big trees, frequency interference from other wireless devices, and weather conditions (rainy, foggy) may affect the video connection and quality.

When conducting a field survey/scan, the recommended flight altitude is around 10-15 meters between 10:00 AM and 3:00 PM on a clear sunny day.

The speed of real-time detection relies on the speed of the processing computer. The higher the specifications of the computing machine, the better. Detection accuracy will primarily depend on the quality of the image training data sets prepared. Lighting conditions due to changing weather and the speed while hovering the camera over the cassava plants may also affect the detection accuracy.

\section{CONCLUSION}

We were able to collect roughly 50,000 raw NIR images of CPD infected cassava from different plantations at San Guillermo Isabela and Diffun, Quirino. We used them as our training and testing datasets. The images collected were verified by our plant pathologist as CPD infected.

Using our developed image processing algorithms, we were able to enhance the features/details of each image on our dataset and further visualize the distinct features of CPD infected cassava leaves, which have a significant impact on our custom CNN model for detecting CPD infected cassavas. Our three custom CPD detection models were fine-tuned using the enhanced NIR images. Each model varies in terms of training duration.

Finally, the study concluded that in terms of image processing speed consideration, the YOLOv4 is best compared to Faster R-CNN inception v2 and SSD MobileNet v2. However, Faster R-CNN inception v2 performs the best compared to the two other models in terms of accuracy. Hence, these two models can be used depending on the purpose of CPD detection. However, since CPD detection is the main purpose of this study, the Faster R-CNN model is recommended for adoption to detect $\mathrm{CPD}$ in a real-time environment.

\section{RECOMMENDATIONS AND FUTURE WORKS}

The researchers intended to improve further the technology focusing on early detection of CPD in cassava plants. These include the increase of the spectral sensitivity of the current camera system; Embedding a system feature to provide highly accurate prediction in root production using a non-invasive 2D (two-dimensional) cassava scanner to other disease and insect-infested cassava plantations. 


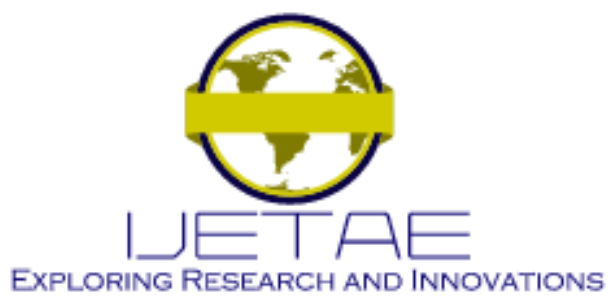

International Journal of Emerging Technology and Advanced Engineering Website: www.ijetae.com (E-ISSN 2250-2459, Scopus Indexed, ISO 9001:2008 Certified Journal, Volume 12, Issue 02, February 2022)

\section{Acknowledgement}

We would like to express our heartfelt appreciation to the Department of Science and Technology (DOST) for funding this project through the Science for Change Program, specifically the CRADLE PROGRAM. The Philippine Council for Agriculture, Aquatic, and Natural Resources Research and Development (PCAARRD) oversees the Agricultural Resources Management Research Division (ARMRD). Finally, to the Isabela State University, Quirino State University, EDCOR, and the Isabela LGU San Guillermo.

\section{REFERENCES}

[1] J. R. R. Uijlings, K. E. A. Van De Sande, T. Gevers, and A. W. M. Smeulders, "Selective search for object recognition," Int. J. Comput. Vis., vol. 104, no. 2, pp. 154-171, 2013.

[2] A. Bochkovskiy, C.-Y. Wang, and H.-Y. M. Liao, "YOLOv4: Optimal Speed and Accuracy of Object Detection," 2020.

[3] Z. Deng, H. Sun, S. Zhou, J. Zhao, L. Lei, and H. Zou, "Multi-scale object detection in remote sensing imagery with convolutional neural networks," ISPRS J. Photogramm. Remote Sens., vol. 145, no. May, pp. 3-22, 2018.

[4] J. Lyu et al., "Extracting the tailings ponds from high spatial resolution remote sensing images by integrating a deep learningbased model," Remote Sens., vol. 13, no. 4, pp. 1-17, 2021.
[5] X. Zhang, Q. Gao, D. Pan, P. C. Cao, and D. H. Huang, "Research on Spatial Positioning System of Fruits to be Picked in Field Based on Binocular Vision and SSD Model," J. Phys. Conf. Ser., vol. 1748, no. 4, 2021.

[6] R. Girshick, J. Donahue, T. Darrell, and J. Malik, "Region-Based Convolutional Networks for Accurate Object Detection and Segmentation,” IEEE Trans. Pattern Anal. Mach. Intell., vol. 38, no. 1, pp. 142-158, 2016.

[7] R. Girshick, "Fast R-CNN," Proc. IEEE Int. Conf. Comput. Vis., vol. 2015 Inter, pp. 1440-1448, 2015.

[8] S. Ren, K. He, R. Girshick, and J. Sun, "Faster R-CNN: Towards Real-Time Object Detection with Region Proposal Networks," IEEE Trans. Pattern Anal. Mach. Intell., vol. 39, no. 6, pp. 1137-1149, 2017.

[9] W. Liu et al., "SSD: Single shot multibox detector," Lect. Notes Comput. Sci. (including Subser. Lect. Notes Artif. Intell. Lect. Notes Bioinformatics), vol. 9905 LNCS, pp. 21-37, 2016.

[10] D. Qiao and F. Zulkernine, "Vision-based Vehicle Detection and Distance Estimation," 2020 IEEE Symp. Ser. Comput. Intell. SSCI 2020, no. June 2021, pp. 2836-2842, 2020.

[11] E. B. Panganiban, I. T. Plata, B. B. Bartolome, A. C. Taracatac, and F. R. E. Labuanan, "Cassava leaf NDVI - Artificial neural network (CaNDVI-ANN): A low cost, portable and non-invasive cassava plant health monitoring device," Int. J. Adv. Trends Comput. Sci. Eng., vol. 8, no. 6, 2019.

[12] P. S. Matthew E. Taylor, "Transfer Learning for Reinforcement Learning Domains: A Survey," J. Mach. Learn. Res., vol. 10, pp. 1633-1685, 2009.

[13] S. J. Pan and Q. Yang, "A survey on transfer learning," IEEE Trans. Knowl. Data Eng., vol. 22, no. 10, pp. 1345-1359, 2010. 